Rev. Elev, Méd. vét. Pays trop., 1968, 21, 2 (219-225).

\title{
Expériences d'embouche des porcs avec mise au pâturage
}

\author{
par J. GILIBERT, P. CAPITAINE, H. SERRES
}

\begin{abstract}
RÉSUMÉ
Deux expériences semblables, une en saison sèche et une en saison des pluies, ont été effecutées pour évaluer l'économie d'aliment concentré qui peut être réalisée en mettant sur du pâturage les porcs à l'engrais, entre les poids de 50 et $100 \mathrm{~kg}$.

Trois traitements alimentaires ont été appliqués à savoir la stabulation permanente hobituelie, la sortie sur pâturage artificiel et la sortie sur pâturage naturel.

Les principaux résultats sont les suivants :

Le pâturage naturel abaisse l'indice de consommation du concentré de 0,3 unité environ, et le pâfurage artificiel de 0,4 unité réalisant une économie de $15 \mathrm{~kg}$ et $20 \mathrm{~kg}$ respecłivement de concentré pour engraisser les porcs du poids de 50 à $100 \mathrm{~kg}$.
\end{abstract}

A Madagascar, et notamment dans le MoyenOuesti, les éleveurs de porcs ont des exploitations de polyculture du type familial. Ils ont à leur disposition un grand nombre de vallons plus ou moins marécageux, appelés lici «Bas-fonds», non cultivés.

Les porcs sont élevés soit en stabulation permanente, soit avec mise au pâturage dans ces bas-fonds pendant la journée.

L'étude présente se propose d'éfudier si la mise au paturage entraîne une diminution de consommation d'aliment concentré, amenant ainsi une économie pour l'élevage.

Dans un premier temps, l'embouche a été étudiée entre les poids de 50 à $100 \mathrm{~kg}$ car les esscis précédents ont montré que les porcelets supportaient mal la mise à l'herbe, et subissaient une infestation massive par les vers parasites, notamment les ascarides.

Une expérience a été réalisée en saison sèche (du 22 avril au 17 juin 1965) et une en saison des pluies (du 24 février au 16 mal 1966).

\section{I. - MATÉRIEL ET MÉTHODES}

Le matériel animal est constitué de porcs Large White, race très répandue à Madagascar, pesant au départ $50 \mathrm{~kg}$.

Dans chacune des expériences trois lots ont été constitués qui recevaient les traitements suivants :

Lot $\mathrm{A}=$ Stabulation permanente, deux repos de concentré par jour (Témoin).

Lot $B=$ Pâturage artificiel matin et soir, un repas de concentré le soir.

Lat $C=$ Pâturage naturel matin et soir, un repas de concentré le soir.

La première expérience a éfé réalisée avec 16 animaux par lot, la seconde aveo 8 animaux par lot.

Le lot A recevait deux repas par jour à 7 heures et à 17 heures.

Les lots $B$ et $C$ sortaient à leur pâturage de $6 \mathrm{~h}$ à $10 \mathrm{~h}$ et de $15 \mathrm{~h}$ à $17 \mathrm{~h}$. A 17 heures, ils recevaient leur repas concentré. 
Durant l'expérience de saison des pluies, le soleil d'été a provoqué des coups de soleil au cours de la période d'adaptation, et les horaires de sortie au. pâturage ont été ramenés aux heures suivantes : $6 \mathrm{~h} 30-9 \mathrm{~h}$ et $16 \mathrm{~h}-17$ heures.

Le pâturage artificiel était constitué par du Brachiaria ruzizıensis de plateau, d'une qualité moyenne au cours de l'essai de saison sèche, car il était à la maturité des graines $(80 \mathrm{~cm}$ de haut). Le principe du pâturage tournant a été utilisé avec clôture électrique, le changement de parcelle étant effectué sans attendre que le pâturage solt rasé ; de cette façon, il n'a pas été détérioré malgré la tendance du porc à fouir la terre.

Le pâturage naturel était fourni par un basfond proche, avec un centre tourbeux, où les animaux étaient en liberté. Pour l'essai de saison des pluies, afin d'éviter la remontée deux fois par jour à la porcherie, une petite porcherie type local sur sol en ciment a été construite en bordure du bas-fond pour les animaux du lot $C$; le protocole d'alimentation ef d'horcires de sorties restant le même que pour les animaux du lot B.

La végétation du bas-fond était haute et abondante, constituée principalement par du Panicum maximum (herbe de Guinée) accompagné d'espèces plus petites et plus fines comme le Leersia hexandra et le Panicum glondulferum, ainsi que des cypéracées dans les points les plus humides.

La quantité de concentré distribuée a été déterminée selon l'appétit des animaux en fixant le temps de repas à vingt minutes pour les lots $B$ et $C$ et à deux foix vingt minutes pour les lots $A$. $\mathrm{Si}$ un lot finissait sa ration en moins de vingt minutes, celle-ci éfait augmentée le lendemain et inversement. Un équilibre a été atteint au cours des quinze jours de période d'adaptation, les porcs ont ensuite assez régulièrement augmenté leur consommation (voir les courbes de consommation).

La composition de la provende distribuée est donnée dans le tableau l.

TABLEAU $N^{\circ} \mathrm{I}$

Composition de la provende

\begin{tabular}{|c|c|c|}
\hline & Lots B et $\mathrm{C}$ & Lot $\mathrm{A}$ \\
\hline Maĩs & $52,5 \mathrm{~kg}$ & $49,5 \mathrm{~kg}$ \\
\hline Manioc & $14,5 "$ & $13,5 "$ \\
\hline Tourteau d'arachide & $8,5 "$ & $8, "$ \\
\hline Son de riz (2/3 fln, $1 / 3$ gros $)$ & $16 \quad "$ & $15 "$ \\
\hline Farine Hareng & 411 & $4 \quad "$ \\
\hline Farine sang local & 311 & 31 \\
\hline Os calciné local & 111 & $1 "$ \\
\hline Farine Stytosanthes (lêgurnineuses) & $0 \quad \mathrm{~g}$ & 511 \\
\hline Coquilles & o $\mathrm{g}$ & $0,5 "$ \\
\hline Sel marin & $0,5 \mathrm{~kg}$ & $0,5 "$ \\
\hline Vitamine A (U.I.) & 400.000 & 400.000 \\
\hline Vitamine $D_{3}$ (U.I.) & 40.000 & 40.000 \\
\hline Sulfate Mn & $4 \mathrm{~g}$ & $4 \mathrm{~g}$ \\
\hline Sulfate $\mathrm{Cu}$ & $0,5 \mathrm{~g}$ & $0,5 \mathrm{~g}$ \\
\hline Sulfate $\mathrm{Zn}$ & $10 \mathrm{~g}$ & $10 \mathrm{~g}$ \\
\hline Vitamine ${ }^{B} 12$ & 98 & $7 \mathrm{~g}$ \\
\hline$T \circ t$ a 1 & $100 \mathrm{~kg}$ & $100 \mathrm{~kg}$ \\
\hline
\end{tabular}


Ces aliments ont été soumis à l'analyse qui a donné les résultats consignés dans le tableau 2 .

TABLEAU $N^{\circ} \mathrm{II}$

Compositton pour $100 \mathrm{~g}$.de matière sèche

\begin{tabular}{|l|c|c|}
\hline & $\begin{array}{c}\text { Aliment } \\
\text { Lot A }\end{array}$ & $\begin{array}{c}\text { Aliment } \\
\text { Lots B et C }\end{array}$ \\
\hline Matiêres minërales & 6,6 & 6,3 \\
\hline Matlères grasses & 5,4 & 5,8 \\
\hline Matiềres azotées & 17,8 & 17 \\
\hline Cellulose brute & 5,2 & 3,6 \\
\hline Extractif non azoté & 65 & 67,3 \\
\hline Insoluble chlorhydrique & 1,8 & 1,8 \\
\hline Phosphore & 0,7 & 0,8 \\
\hline Calclum & 0,65 & 0,6 \\
\hline
\end{tabular}

\section{II. - EXPÉRIENCE DE SAISON SÈCHE}

\section{A. - Croissance.}

10 Pords moyens ef craissances (tableau 3).

\section{$2^{\circ}$ Observations.}

Une différence significatıve au seuil 0,05 apparaît entre le lot $A$ et lots $B$ et $C$. Aucune différence n'apparaît entre les lots $B$ et $C$.

Les animaux en stabulation permanente ont eu une croissance plus rapide que celle des animaux allant au pâturage, leur supériorité étant de sept kilos environ en 57 jours d'expérience. Ceci revient à dire que les animaux du lot $A$ ont atteint le poids de commercialisation une semaine avant les animaux des lots $B$ et $C$.

$3^{\circ}$ Croits quotidiens moyens en grommes por semane ef par onimal (tableau 4).

II esł à noter que les variations de croissances hebdomadaires sont parallèles pour les trois

TABLEAU $N^{\circ}$ III

Poids moyens des lots en dêbut d'expêrience le 22.4 et en fin d'expérlence 1e 17.6. (Lats de 16 pores)

\begin{tabular}{|c|c|c|c|}
\hline & A & B & C \\
\hline Polds au 22/4 & $54,25 \pm 1,06$ & $55,19+1,26$ & $54,46 \pm 0,9$ \\
\hline Poids au $17 / 6$ & $102,98 \pm 1,93$ & $96,76 \pm 1,72$ & $95,06 \pm 1,82$ \\
\hline Gain & 48,73 & 41,57 & 40,60 \\
\hline
\end{tabular}

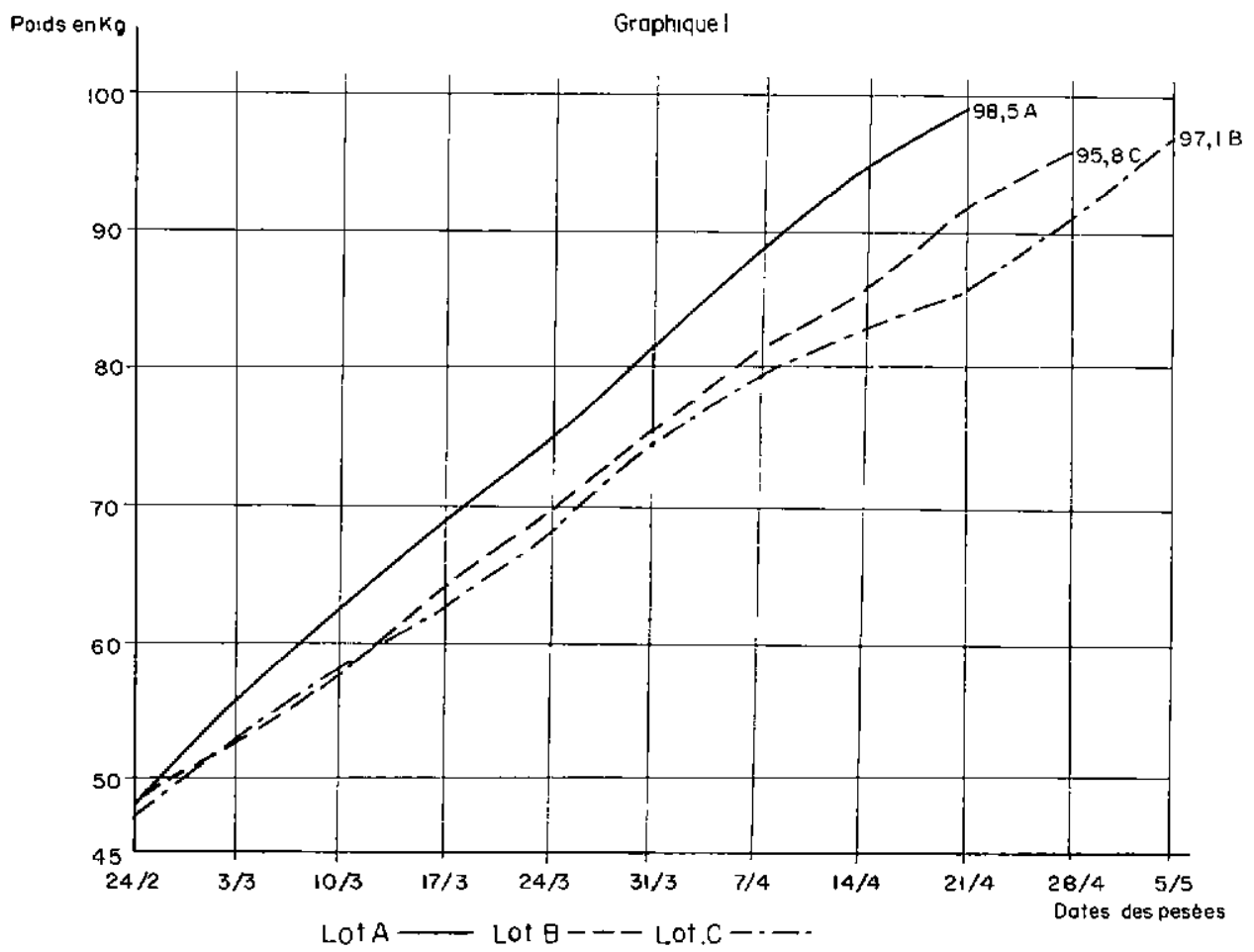


TABLEAU N ${ }^{\circ} \mathrm{IV}$

Crô̂ts quotidiens moyens en grammes par semaine et par andmal

\begin{tabular}{|c|c|c|c|c|c|c|c|c|}
\hline & lère & $2 \tilde{e}$ & 3 อ̀ & $4 \mathrm{e}$ & $5 e ̄$ & 6๕̀ & $7 e ̀$ & $8 \overline{\mathrm{e}}$ \\
\hline Lot $A$ & 1.007 & 523 & 805 & 988 & 787 & 1.054 & 798 & 987 \\
\hline Lot B & 1.020 & 474 & 551 & 814 & 573 & 847 & 763 & 890 \\
\hline Lot $\mathrm{C}$ & 1.030 & $42 B$ & 650 & 847 & 591 & 838 & 666 & 744 \\
\hline
\end{tabular}

lots, ce qui est un phénomène assez constant dans les expériences d'alimentation.

On en conclut que les animaux des lots $B$ et $C$ se sont accoutumés assez facilement à leur mise au pâturage, bıen qu'il y ait changement de mode de vie (plein air) et changement d'alimentation (herbe verte).

\section{B. - Consommation.}

10 Consommation de provende por jour ef par animal (moyennes par lot) (tableau 5).

Les animaux du lot $A$ présentent une augmentation de la consommation régulière ef classique pour les porcs élevés uniquement d̀ la provende.

Les animaux $B$ ef $C$ ont une variation de la consommation parallèle. La consommation reste stationnaire pendant les premières semaines, puis monte ensuite régulièrement.

$2^{\circ}$ Gain de poids total ef indices de consommation (tableau 6).
Les indices de consommation ont été calculés par rapport à la provende consommée sans tenir compte de l'herbe. Le pâturage représente du point de vue économique une quantité négligeable comme il sera exposé plus loin dans les bilans financiers.

Des différences significatives apparaissent entre les lots A d'une part, $B$ et $C$ d'autre part.

La mise au pâturage des porcs entraîne donc une économie considérable de provende, notamment pour le lot sur pâturage artificiel.

Remarque : Les animaux allant au pâturage présentaient une conformation netfement meilleure que celle de ceux qui sont demeurés en stabulation. Bien que les mesures d'épaisseur du lard n'aient pas alors été effectuées, on peut affirmer après avoir vu les animaux abattus, que les animaux de pâturage présentaient moins de gras.

\section{TABLEAU $N N^{\bullet}$}

Consonmation de provende par jour et par animal (moyennes par 1ot)

\begin{tabular}{|l|c|c|c|c|c|c|c|c|}
\hline & 1 er & 2 è & $3 \tilde{\mathrm{e}}$ & $4 \mathrm{e}$ & $5 \check{\mathrm{e}}$ & $6 \tilde{\mathrm{e}}$ & 7 è & $8 \mathrm{e}$ \\
\hline Lot A & 2,35 & 2,45 & 2,65 & 2,90 & 3,13 & 3,37 & 3,60 & 3,61 \\
\hline Lot B & 2,01 & 1,90 & 1,97 & 2,14 & 2,24 & 2,36 & 2,50 & 2,67 \\
\hline Lot C & 1,98 & 1,84 & 1,98 & 2,14 & 2,30 & 2,46 & 2,53 & 2,64 \\
\hline
\end{tabular}

TABLEAU N ${ }^{\circ} \mathrm{VI}$

\begin{tabular}{|c|c|c|c|}
\hline & Lot A & Lot B & Lot C \\
\hline Gain de poids total & 779,7 & 665,2 & $.649,6$ \\
\hline Aliment consomer & 2700,1 & 1995,7 & 2006,9 \\
\hline Indice consomation & 3,46 & 3,00 & 3,08 \\
\hline
\end{tabular}


1II. - EXPÉRIENCE DE SAISON DES PLUIES

\section{A. Croissance.}

10 Poids moyens ef croissances (tableau 7).

\section{TABLEAU $\mathrm{N}^{\circ} \mathrm{VII}$}

Poids uloyens des lots en début d'expêrience le $24 / 2$ et en fin d'expérience à partir du $21 / 4$ (lots de 8 porcs)

\begin{tabular}{|c|c|c|c|}
\hline & A & B & C \\
\hline $24 / 2$ & 48,1 & 47,5 & 48,2 \\
\hline $21 / 4$ & 98,5 & 86,1 & 92,0 \\
\hline Croissance & $50,4 \pm 3,8$ & $38,6 \pm 2,5$ & $43,8 \pm 3,0$ \\
\hline
\end{tabular}

20 Observations : Les lots ont été retirés de l'expérience quand ils atteignaient cent kilos à des dates différentes car ils ont eu des vitesses de croissance inégales.
Entre les lots $A, B$ et $C$ comprenant chacun 8 animaux, il apparaît des différences significatives au seuil 0,05 entre les lots $A$ et $B$, ef non entre les lots $A$ et $C$ ef $B$ et $C$.

Les animaux en stabulation permanente ont eu une croissance plus rapide que ceux sortant au pâturage, leur supériorité étant de 7 à $12 \mathrm{~kg}$ : en 56 jours d'expérience, ils atteignent le poids de vente entre une et deux semaines avant les autres, ce qui confirme les résultats de l'expérience de saison sèche.

30 Croits quotidiens moyens en grammes por semaine et por animal.

Les variations de croissances hebdomadaires sont parallèles pour les lots $A$ et $B$, ce qui entraîne les mêmes remarques que pour l'expérience de saison sèche.

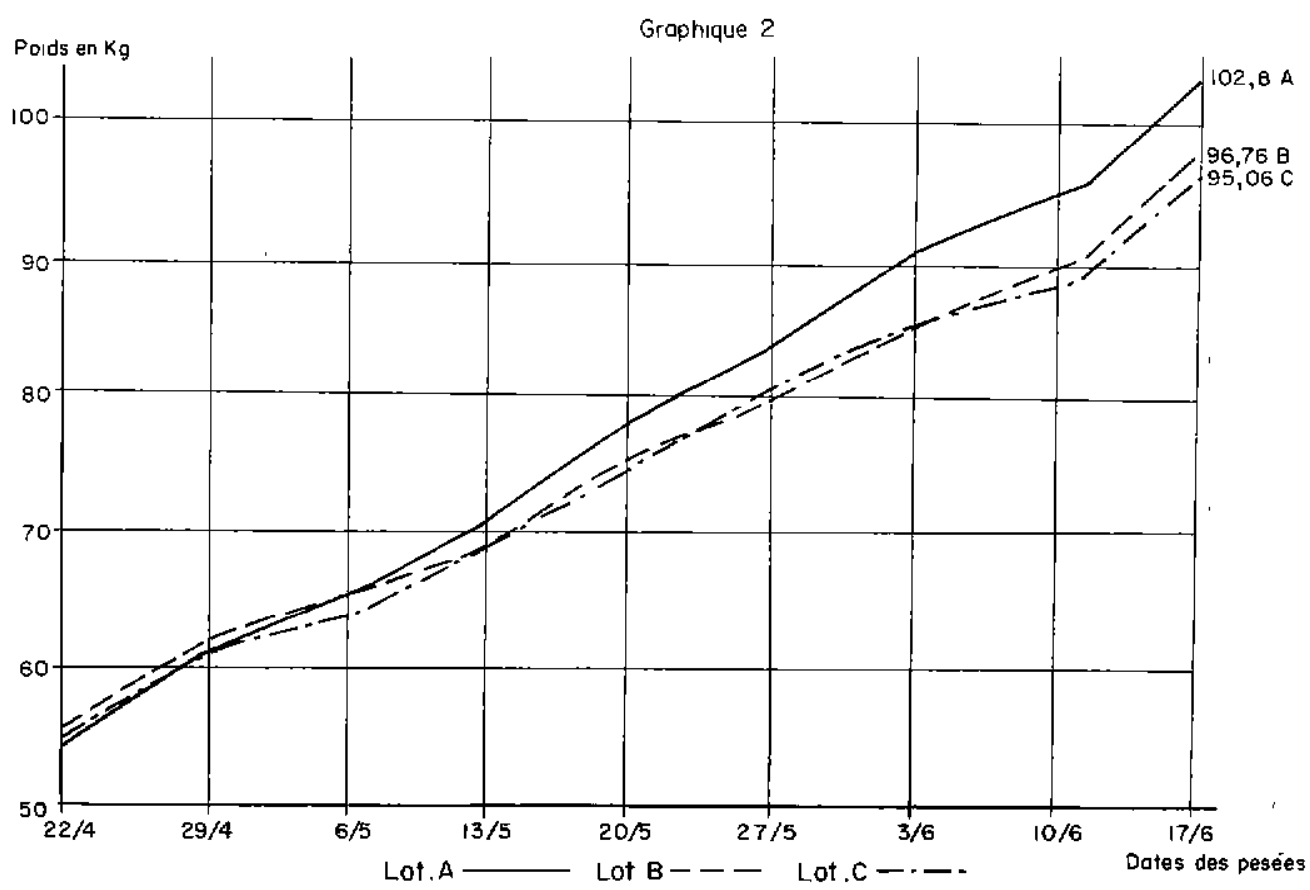

TABLEAU $\mathbb{N}^{\circ}$ VIII

Croîts quotidiens moyens en grammes par semaine et par animal

\begin{tabular}{|c|c|c|c|c|c|c|c|c|c|c|}
\hline & lex & $2 \mathrm{è}$ & $3 \grave{e}$ & $4 \mathrm{è}$ & $5 \mathrm{e}$ & $6 \mathrm{è}$ & $7 \mathrm{è}$ & $8 \mathrm{e}$ & $9 \mathrm{e}$ & $10 \mathrm{e}$ \\
\hline Lot A & 1.128 & 985 & 842 & $82 \mathrm{~B}$ & 1.000 & 957 & 871 & 585 & & \\
\hline Lot B & 742 & 742 & 657 & 814 & 871 & 700 & 500 & 485 & 700 & $\mathrm{~B} 71$ \\
\hline -Lot C & 642 & 714 & 914 & 757 & 871 & 885 & 628 & 842 & 542 & \\
\hline
\end{tabular}


TABLEAU $N^{\circ} \mathrm{IX}$

Consonmation moyenne de provende par jour et par animal

\begin{tabular}{|c|c|c|c|c|c|c|c|c|c|c|}
\hline & ler & $2 \mathrm{e}$ & $3 \mathrm{e}$ & $4 \mathrm{e}$ & $5 \mathrm{e}$ & $6 \mathrm{e}$ & $7 \mathrm{e}$ & $8 \mathrm{e}$ & $9 \mathrm{e}$ & $10 \mathrm{e}$ \\
\hline Lot A & 2,5 & 2,8 & 3,1 & 3,1 & 3,1 & 3,3 & 3,2 & 2,9 & & \\
\hline Lot B & 1,6 & 2,0 & 2,1 & 2,3 & 2,3 & 2,3 & 2,2 & 2,1 & 2,2 & 2,2 \\
\hline Lot C & 1,9 & 2,1 & 2,4 & 2,5 & 2,4 & 2,6 & 2,6 & 2,7 & 2,2 & \\
\hline
\end{tabular}

TABLEAU $N^{\circ} \mathrm{X}$

Les indices de consommation ont êté calculës comme précédenment

\begin{tabular}{|c|c|c|c|}
\hline & Lot $A$ & Lot $B$ & Lot $\mathrm{C}$ \\
\hline Gain de polds total & 421,6 & 431,5 & 437,4 \\
\hline Allment consommé & 1407,2 & 1295,2 & 1352,6 \\
\hline Indice consommation & 3,34 & 3,00 & 3,09 \\
\hline
\end{tabular}

Pour le lot $C$, les variations ne sont pas parallèles, ce qui est probablement dô au fait que le lot $C$ étalt dans une porcherie séparée et dans une ambiance différente (situation en bas-fonds, porcherie de style local).

\section{B. - Consommation.}

10 Consommation moyenne de provende par jour et par animal.

La consommation de provende n'a pas la même allure que lors de l'expérience précédente: elle présente ici une augmentation régulıère pendant les six premıères semaines de l'expérience.

Pour les lots $A$ et $C$, on constate une dimınution de consommation de provende en fin d'engraissement.

La même remarque que pour l'expérience de saison sèche peut être faite : les anımaux allant au pâturage étalent mieux conformés et moins gras.

$2^{\circ}$ Gain de poids total et indices de consommation (tablecu 10).

Des différences apparaissent entre les lots $A, B$ et $C$.

Les résultats en saison des pluies présentent donc une remarquable concordance avec ceux de saison sèche, et ceci malgré un temps de pâturage inférieur (tableau 11).
TABLEAU $\mathrm{N}^{\circ} \mathrm{XI}$

\begin{tabular}{|c|c|c|}
\hline & $\begin{array}{c}\text { Saison } \\
\text { seche }\end{array}$ & $\begin{array}{c}\text { Saison } \\
\text { des pluies }\end{array}$ \\
\hline $\begin{array}{c}\text { Indice consonmation } \\
\text { Lot A }\end{array}$ & 3,46 & 3,34 \\
\hline $\begin{array}{c}\text { Indice consommation } \\
\text { Lot B }\end{array}$ & 3,00 & 3,00 \\
\hline $\begin{array}{c}\text { Indice consommation } \\
\text { Lot C }\end{array}$ & 3,08 & 3,09 \\
\hline
\end{tabular}

Il semble que les porcs au pâturage artificiel consomment davantage d'herbe qu'au pâturage naturel, ce qui diminue la consommation de provende, améliore l'indice de consommation, mais dıminue en même temps la vitesse de croissance.

\section{IV. - INTÉRÊT FINANCIER}

Pour l'engraissement des porcs entre 50 et $100 \mathrm{~kg}$, la mise au pâturage naturel abaisse l'indice de consommation de la provende de 0,3 environ par rapport à la stabulation permanente ef la mise au pâturage artificiel l'abaisse de 0,4 environ.

Les provendes utilisées pour les différents lots coûtent le même prix dans le commerce, à savoir 20 FMG le kg.

Le pâturage naturel économise $15 \mathrm{~kg}$ de provende, soit 300 FMG. 
Le pâturage artificiel économıse $20 \mathrm{~kg}$ de provende, soit 400 FMG.

Ce gain n'est pas négligeable pour le paysan lorsqu'on sait que le salaire officiel d'un manceuvre journalier est de 130 FMG par jour.

Un éleveur engraissant un lot de dix porcs aura gagné trois mille FMG par le simple fait de laisser ses animaux dans un bas-fond matin et soir.

Des mensurations de carcasses n'ont pu être effectuées sur ces animaux à la fin de l'expé- rience ; cependant, par jugement à l'œil sur les animaux vivants, il est apparu nettement que les anımaux sortant au pâturage étaient nettement moins gras que ceux de stabulation libre, mieux conformés et plus alertes. La méthode paraît à recommander pour la production de géniteurs.

\section{Institut d'Elevage}

et de Médecine vétérinare des Pays tropicaux.

Région de Recherches vétérinoires ef zootechniques de Madagascar d Tananarive.

\section{SUMMARY}

\section{Experiments with fattening pigs on pasture}

Two similar experiments, one in the dry season and the other in the rainy season, were made in order to assess the saving of concentrate made possible by the fattening on pasture of pigs between 50 and $100 \mathrm{~kg}$.

Three dietary regimes were applied :

1. Permanent housıng ; 2. Grazing on leg ; 3. Grazing on permanent pasture.

The moin results are as follows :

The consumption of concentrate 15 reduced on permanent pasture by about 0,3 unit, and on seeded pasture by 0,4 unit, saving respectively $15 \mathrm{~kg}$ and $20 \mathrm{~kg}$ of concentrate for the fattening of pigs weighıng belween 50 and $100 \mathrm{~kg}$.

\section{RESUMEN}

\section{Ensayos de engorde a pasio de los cerdos}

Se efectuaron dos ensayos semejantes, el uno durante la estación seca y el otro durante la estación de las lluvias, para evaluar la economia de pienso concentrado pudiendo realizarse con el pastoreo de los cerdos de engorde pesando de 50 a $100 \mathrm{~kg}$. Se aplicaron los tresmodos de alimentación siguientes : en establo permanente habitual, en pastoreo sobre pastos artıficiales y naturales.

Los principales resultados son los siguientes : El pasto natural disminuye el indice de consumo del concentrado de hasta 0,3 unidad, y el pasto artificial de 0,4 unidad, realizando una economia de concentrado de 15 y $20 \mathrm{~kg}$ para el engorde de los cerdos del peso de 50 a $100 \mathrm{~kg}$. 